\title{
Sex Difference in the Morningness-Eveningness Preference in Student and Hospital Nurse Samples
}

\author{
Key words: Morningness-eveningness-Shift work-Sleep-wake habits-Question- \\ naire study-Sex difference
}

Recently, interindividual differences in tolerance to shift work have become an increasing interest in chronobiological shift work studies. ${ }^{1)}$ A number of factors have been investigated ${ }^{2)}$ such as sex, age, general health status, quality and quantity of sleep, internal desynchronization between body temperature and sleep-wake cycle, morningness-eveningness, introversion-extroversion, job satisfication, etc. Among them, the morningness-eveningness (M-E) preference has received much attention since the publication of the M-E questionnaire in an English version of the report by Horne and Östberg. ${ }^{3)}$ This questionnaire was translated into other languages and its validity has been confirmed by many authors. ${ }^{4-10}$ ) However, the sample populations were students in most of these studies, ${ }^{5-9)}$ so that it remains to be determined whether there are differences between students and other populations (for example, shift workers). The present study was carried out to compare the M-E preference, with respect to sex difference, between students and shift-working hospital nurses using a Japanese version of the M-E questionnaire.

An English version of the M-E questionnaire of Horne and Östberg ${ }^{3)}$ was translated into Japanese, and this questionnaire was administered to 248 students (110 males and 138 females, 20.9 \pm 2.8 years old, mean \pm SD) and 138 hospital nurses (all females, 28.3 \pm 8.6 years old) working in three shifts (day, evening and night shift) on a short-term (2-3 days) rotation system. The M-E preference was assessed by a single M-E score based on this self-assessment questionnaire consisting of 19 questions. Five categories were formed on the basis of the scoring criteria of Horne and Üstberg"): extremely morning type (score range 70-86), moderately morning type (59-69), intermediate type (42-58), moderately evening type (31-41) and extremely evening type (16-30). In addition, a sleep-wake habit questionnaire to determine the times of going to bed and waking up for the past three consecutive days as well as the recent quality of sleep (easiness to falling asleep, interruption of sleep, self-estimation of soundness of sleep) was administered at the same time. The questionnaire was administered in June.

Figure 1(a) and 1(b) show the distributions of scores for both sexes in the student sample. The scores did not differ significantly from a normal distribution in either sex (Kolmogorov-Smirnov test). There was a statistically significant difference in mean scores between male $(42.6 \pm 10.5$, mean $\pm S D)$ and female $(48.8 \pm 8.2)$ students. Figure 1 (c) shows the distribution of scores for hospital 

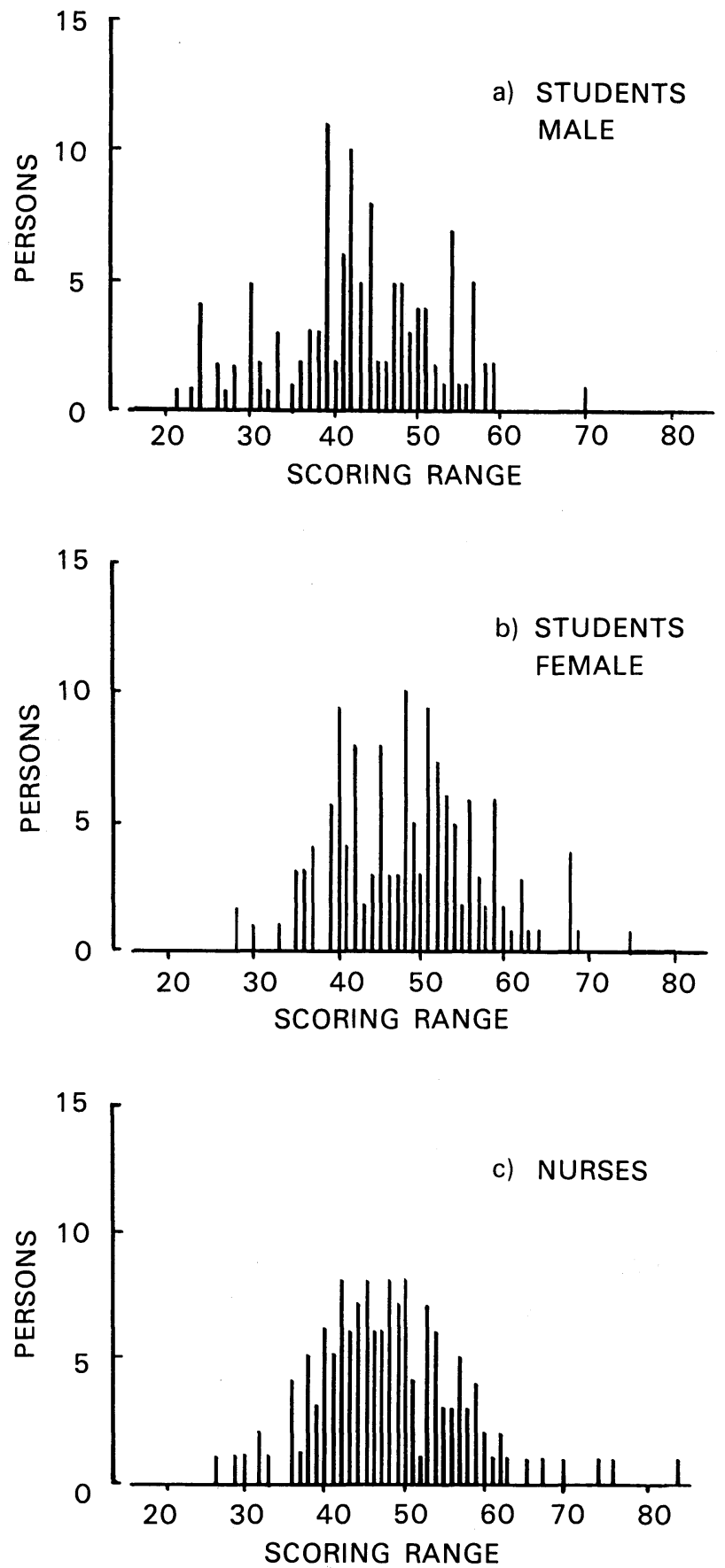

Fig. 1. Distributions of morningness-eveningness scores in students (a : male, b : female) and hospital nurses (c) 
Table 1. Bedtime, waking time and sleep length in each chronotype of both sexes in students (Mean \pm SD)

\begin{tabular}{clccc}
\hline & & Morning type & Intermediate type & Evening type \\
\hline Bedtime & male & $23.5 \pm 0.8(\mathrm{n}=4)$ & $0.8 \pm 1.3(\mathrm{n}=71)$ & $2.0 \pm 1.4(\mathrm{n}=50)$ \\
(o'clock) & female & $0.2 \pm 0.7(\mathrm{n}=22)$ & $0.4 \pm 1.1(\mathrm{n}=94)$ & $1.0 \pm 1.2(\mathrm{n}=33)$ \\
Waking time & male & $7.1 \pm 1.0$ & $7.5 \pm 1.5$ & $8.2 \pm 1.7$ \\
$\quad$ (o'clock) & female & $6.5 \pm 1.2$ & $6.5 \pm 1.0$ & $7.0 \pm 0.6$ \\
Sleep length & male & $7.2 \pm 0.7$ & $6.7 \pm 1.3$ & $6.2 \pm 2.0$ \\
(hours) & female & $6.5 \pm 1.2$ & $6.1 \pm 1.2$ & $6.0 \pm 1.0$ \\
\hline
\end{tabular}

nurses $(47.7 \pm 9.4), 77 \%$ of whom were working a normal day shift on the day of inquiry. The difference in scores between hospital nurses and female students was not statistically significant, while the difference between hospital nurses and male students was significant $(\mathrm{p}<0.01)$. Table 1 shows means and standard deviations of sleep parameters for the morning, the intermediate and the evening types in the student sample. There was a tendency for both bedtime and waking time to be later in the evening type than in the morning type and for sleep length to be shorter in the former than the latter. Significant correlations were observed between scores and sleep parameters of mid-sleep time, bedtime and waking time (Table 2). Sleep parameters in the nurse sample were not analyzed since both bedtime and waking time in the evening and night shift were hard to evaluate.

The present study revealed that the M-E scores did not differ from the normal distribution in either student or hospital nurse samples. These results agree with most of the previous reports in various languages (Swedish, ${ }^{4)}$ English, ${ }^{5)}$ and Dutch $^{6)}$ ), all of which had nearly the same sample size. However, particular attention should be given to the shape of the distribution of M-E scores. Meccaci and $\mathrm{Zani}^{10)}$ reported that the score distribution on an Italian version of $\mathrm{M}-\mathrm{E}$ questionnaire negatively skewed and differed from a normal distribution. In a recent review, ${ }^{11)}$ Kerkhof discussed this discrepancy in the shape of the score distribution and pointed out the importance of socio-cultural differences in various countries. He advised that the M-E questionnaire be adapted to the particular countries and cultures if necessary. Such an adaptation was done for Swedish ${ }^{4}$ and Dutch ${ }^{6)}$ versions, and the scores were distributed normally for both studies. In the Japanese questionnaire used in this study, no modification seemed to be

Table 2. Correlation coefficients between morningness-eveningness scores and four variables of sleep-wake habits in students $(n=248)$

\begin{tabular}{lc}
\hline Bedtime & $-0.464^{* *}$ \\
Waking time & $-0.398^{* *}$ \\
Mid-sleep time & $-0.457^{* *}$ \\
Sleep length & 0.051 \\
\hline$* *: \mathrm{P}<0.01$ &
\end{tabular}


necessary.

The significant correlation between M-E scores and sleep parameters also supports previous reports. ${ }^{5,6,9)}$ Further attention should be paid to the result that there was no significant difference in mean M-E scores between hospital nurses and female students, although a significant difference was found between male and female students. It appears that the irregular working schedule did not affect the morningness-eveningness preference in the hospital nurse sample. This is in contrast to the study of Meceaci and Zani, ${ }^{10)}$ which found a significant difference in mean M-E scores between white collar workers with regular working schedules and student samples. It is important to note that these authors did not refer to any sex difference. As was shown in this report as well as the report by Ishihara et al., ${ }^{9)}$ female populations seem to be more the morning type than male populations. Therefore, sex difference should be taken into account for morningnesseveningness studies on workers.

In brief, this study suggested that an irregular working schedule was less important in influencing the morningness-eveningness preference than sex in the populations investigated. Further investigation is needed on other populations to elucidate the influence of sex and occupation on the morningness-eveningness preference.

\section{REFERENCES}

1) Smolensky MH, Paustenbach DT, Scheving LE. Biological Rhythms, Shift Work and Occupational Health. In: Crally L, Crally L, eds. Industrial Hygiene and Toxicology. 2nd Ed. Vol. 3b. London, New York: John Wiley \& Sons, 1985; 156-312.

2) Smolensky MH. Chronoepidemiology of Shift Work. In: Reinberg A, Vieux N, Andlauer P, eds. Night and Shift Work. Biological and Social Aspects. Oxford: Pergamon Press, 1981; 51-65.

3) Horne JA, Östberg O. A self-assessment questionnaire to determine morningnesseveningness in human circadian rhythms. Int J Chronobiol 1976; 4: 97-110.

4) Torsvall L, Äkerstedt T. A diurnal type scale. Construction, consistency and validation in shift work. Scand J Work Env Health 1980; 614: 283-90.

5) Posney TB, Ford JA. The morningness-eveningness preference of college students as measured by the Horne and Östberg questionnaire. Int J Chronobiol 1981; 7: 141-4.

6) Kerkhof GA. A Dutch-language questionnaire for the selection of morning and evening type individuals (In Dutch). Nederlands Tijdschrift voor de Psychologie 1984; 39: 281-94.

7) Hildebrandt G, Stratmann I. Circadian system responses to night work in relation to the individual circadian phase position. Int Arch Occup Env Health 1977; 43: 73-83.

8) Fröberg JE. Twenty-four hour patterns in human performance, subjective and physiological variables between morning and evening active subjects. Biol Psychol 1977; 5: $119-34$.

9) Ishihara K, Miyashita A, Inugami M, Fukuda K, Yamazaki K, Miyata Y. The results of investigation by the Japanese version of Morningness-Eveningness Questionnaire. Jap J Psychol 1986; 57: 87-91.

10) Mecacci I, Zani A. Morningness-eveningness preferençes and sleep-waking diary data of morning and evening types in student and worker samples. Ergonomics 1983; 26; 
1147-53.

11) Kerkhof GA. Inter-individual differences in the human circadian system: A review. Biol Psychol 1985; 20: 83-112.

Department of Hygiene, Faculty of Medicine

Yutaka MOTOHASHI

Tokyo Medical and Dental University

5-45, 1-chome, Yushima, Bunkyo-ku,

Tokyo, 113, Japan

(Received June 29, 1988 and in revised form September 8, 1988) 